\title{
ON THE SUM OF POWERS OF SQUARE MATRICES
}

\author{
Dinesh J. Karia, Kailash M. Patil and H. P. Singh
}

Abstract. Given a $2 \times 2$ matrix $A$, we obtain the formula for sum of $A^{n},(n \in \mathbb{Z})$, using its trace and determinant only; this includes the negative powers in the case of a nonsingular matrix too. Here we mean by sum, the sum of all the entries of the matrix. Various special cases arising out of values of trace and determinant are discussed and as an application we also derive MarcusNewman inequality proved by D. London. $2 \mathrm{su}\left(A^{3}\right) \geqslant \operatorname{su}(A) \operatorname{su}\left(A^{2}\right)$, for all $A \in \mathscr{F}_{2} \cap \mathscr{M}_{2}^{+}$.

Mathematics subject classification (2010): Primary 15A24, Secondary 15A45.

Keywords and phrases: Sum of elements of a matrix, power of a matrix, nonsingular matrix, symmetric matrix, trace, determinant.

\section{REFERENCES}

[1] H. KankAanp ̈̈̈̈ And J. K. Merikoski, Two inequalities for the sum of elements of a matrix, Linear and Multilinear Algebra 18 (1) (1985), 9-22.

[2] D. London, Inequalities in quadratic forms, Duke Math. J. 33 (1966), 511-522.

[3] D. London, Two Inequalities in nonnegative symmetric matrics, Pacific J. Math 16 (1966), 515-536.

[4] M. Marcus And M. Newman, The sum of the elements of the powers of a matrix, Pacific J. Math 12 (1962), 627-635.

[5] J. K. MERIKOSKI, On the trace and the sum of elements of a matrix, Linear algebra and its applications 60 (1984), 177-185.

[6] L. Seymour, Note on graphs and matrix inequalities, The Amer. Math. Monthly 92 (1) (1985), 277-280. 